Swarthmore College

Works

Physics \& Astronomy Faculty Works

Physics \& Astronomy

6-1-2008

\title{
Flow Dynamics And Plasma Heating Of Spheromaks In SSX
}

Michael R. Brown

Swarthmore College, doc@swarthmore.edu

C. D. Cothran

David H. Cohen

Swarthmore College, dcohen1@swarthmore.edu

Jason Alexander Horwitz , '07

Vernon Hampden Chaplin , '07

Follow this and additional works at: https://works.swarthmore.edu/fac-physics

Part of the Astrophysics and Astronomy Commons, and the Physics Commons

Let us know how access to these works benefits you

\section{Recommended Citation}

Michael R. Brown; C. D. Cothran; David H. Cohen; Jason Alexander Horwitz , '07; and Vernon Hampden Chaplin , '07. (2008). "Flow Dynamics And Plasma Heating Of Spheromaks In SSX". Journal Of Fusion Energy. Volume 27, Issue 1-2. 16-19. DOI: 10.1007/s10894-007-9097-y https://works.swarthmore.edu/fac-physics/22

This work is brought to you for free by Swarthmore College Libraries' Works. It has been accepted for inclusion in Physics \& Astronomy Faculty Works by an authorized administrator of Works. For more information, please contact myworks@swarthmore.edu. 


\title{
Flow dynamics and plasma heating of spheromaks in SSX
}

\author{
M. R. Brown* and C. D. Cothran, D. Cohen, J. Horwitz, and V. Chaplin \\ Department of Physics and Astronomy \\ Center for Magnetic Self Organization \\ Swarthmore College \\ Swarthmore, PA 19081-1397
}

(Dated: April 5, 2007)

\begin{abstract}
We report several new experimental results related to flow dynamics and heating from single dipole-trapped spheromaks and spheromak merging studies at SSX. Single spheromaks (stabilized with a pair of external coils, see Brown, Phys. Plasmas 13102503 (2006)) and merged FRC-like configurations (see Brown, Phys. Plasmas 13, 056503 (2006)) are trapped in our prolate $(R=$ $0.2 m, L=0.6 \mathrm{~m}$ ) copper flux conserver. Local spheromak flow is studied with two Mach probes $\left(r_{1} \leq \rho_{i}, r_{2} \geq \rho_{i}\right)$ calibrated by time-of-flight with a fast set of magnetic probes at the edge of the device. Both Mach probes feature six ion collectors housed in a boron nitride sheath. The larger Mach probe will ultimately be used in the MST reversed field pinch. Line averaged flow is measured by ion Doppler spectroscopy (IDS) at the midplane. The SSX IDS instrument measures with $1 \mu \mathrm{s}$ or better time resolution the width and Doppler shift of the $C_{I I I}$ impurity (H plasma) $229.7 \mathrm{~nm}$ line to determine the temperature and line-averaged flow velocity (see Cothran, RSI 77, 063504 (2006)). We find axial flows up to $100 \mathrm{~km} / \mathrm{s}$ during formation of the dipole trapped spheromak. Flow returns at the wall to form a large vortex. Recent high-resolution IDS velocity measurements during spheromak merging show bi-directional outflow jets at $\pm 40 \mathrm{~km} / \mathrm{s}$ (nearly the Alfvén speed). We also measure $T_{i} \geq 80 \mathrm{eV}$ and $T_{e} \geq 20 \mathrm{eV}$ during spheromak merging events after all plasma facing surfaces are cleaned with helium glow discharge conditioning. Transient electron heating is inferred from bursts on a 4-channel soft x-ray array. The spheromaks are also characterized by a suite of magnetic probe arrays for magnetic structure $\mathbf{B}(r, t)$, and interferometry for $n_{e}$. Finally, we are designing a new oblate, trapezoidal flux conserver for FRC studies. Equilibrium and dynamical simulations suggest that a tilt-stable, oblate FRC can be formed by spheromak merging in the new flux conserver. Keywords: spheromak, flow, heating
\end{abstract}

\section{INTRODUCTION}

Magnetic reconnection is the engine driving the dynamics in spheromak formation, relaxation, and merging $[1,2]$. During reconnection, magnetic energy is rapidly converted to electron and ion heat, as well as plasma flow. Spheromak dynamics have been studied at the Swarthmore Spheromak Experiment (SSX) in a number of geometries [3-6]. We have recently been studying spheromak merging in a prolate $0.4 \mathrm{~m}$ diameter, $0.6 \mathrm{~m}$ length, $3 \mathrm{~mm}$ wall copper flux conserver at SSX. Merging of a pair of counter-helicity spheromaks generates magnetic reconnection dynamics at the midplane. Injection of a single spheromak into a vacuum dipole magnetic field generates a quiescent, axisymmetric toroidal structure that is stable for the lifetime of the object, despite the elongated flux conserver.

Local and global magnetic structure of SSX spheromaks has been studied with up to 600 individual internal magnetic probes operated simultaneously at 1.25 $\mathrm{MHz}$ using a multiplexer system [7]. Line averaged electron density is monitored with a quadrature $\mathrm{HeNe}$ laser interferometer [8]. We can scan density in a range $n_{e}=1-10 \times 10^{14} \mathrm{~cm}^{-3}$. Line averaged electron temperature is inferred from a model fit to data from a soft

\footnotetext{
${ }^{*}$ Electronic address: doc@swarthmore.edu
}

x-ray array.

In addition, line averaged ion flow and temperature $T_{i}$ at the midplane is monitored with a $1.33 \mathrm{~m}$ ion Doppler spectrometer (IDS) for this configuration. We achieve high spectral resolution (0.0075 $\mathrm{nm}$ per pixel) with an Echelle grating operating at 25 th order [9]. We achieve high temporal resolution by using a 32 channel photomultiplier tube array. The SSX IDS instrument measures with $1 \mu s$ or better time resolution the width and Doppler shift of the $C_{I I I}$ impurity (H plasma) $229.7 \mathrm{~nm}$ line to determine the temperature and line-averaged flow velocity during spheromak trapping. The instrument design temperature is approximately $3 \mathrm{eV}$ for $C$ emission lines. Velocity resolution is about $5 \mathrm{~km} / \mathrm{s}$, corresponding to approximately $0.05 v_{A}$. Line averaged IDS measurements are corroborated locally with a Mach probe.

In section II, measurements of the reconnectiondriven outflow are discussed (both spectroscopic and probe measurements). In section III, measurements of reconnection-driven heating (ions and electrons) is presented. A summary is presented in section IV.

\section{RECONNECTION OUTFLOW}

The SSX apparatus uses opposing, coaxial magnetized plasma guns at either end of a cylindrical copper flux conserver to produce spheromaks of either handedness (see figure 1). Several different flux conservers have been 
used at SSX over the years with diameters varying between 0.17 and $0.5 \mathrm{~m}$. The present flux conserver diameter is $0.40 \mathrm{~m}$ with a length of $0.61 \mathrm{~m}$ (aspect ratio or elongation of 1.5 and $L / R=3$ ). Spheromak merging drives magnetic reconnection at the midplane. We expect unmagnetized plasma to be expelled from both sides of the reconnection region at the Alfvén speed. We have made line-averaged measurements of this process with ion Doppler spectroscopy and local measurements with Mach probes.

\section{A. Ion Doppler spectroscopy}

In figure 2 , we show a lineshape from $C_{I I I}$ emission measured during a single reconnection event. The chord for this sequence is across the full $0.4 \mathrm{~m}$ diameter at the midplane as shown in figure 1. Motion both towards and away from the observer is evident. Simultaneous bidirectional outflow with velocities $\pm 40 \mathrm{~km} / \mathrm{s}$ is clearly evident at about $40 \mu \mathrm{s}$ [10]. An outflow jet of $40 \mathrm{~km} / \mathrm{s}$ corresponds to about $0.4 V_{A}$ in SSX. Note that since the process is dynamic and fully $3 \mathrm{D}$, we observe bi-directional jets in only 1 in 5 shots.

\section{B. Mach probe}

A Mach probe is essentially a directional electrostatic ion collector measuring upstream and downstream ion current simultaneously. SSX Mach probes feature 3 pairs of opposing tungsten collectors housed in boron nitride sheath. The Mach number measured by the probe is equal to $M=K \ln \left(I_{u p} / I_{\text {down }}\right)$ (where $K$ is a proportionality constant). While $a b$ initio calculation of the proportionality constant $K$ is both complicated and controversial [11], SSX Mach probes are absolutely calibrated using a time-of-flight technique. Using an array of edge magnetic probes, we track the trajectory of single spheromaks ejected from one gun or the other. We determine a mean calibration constant for a given probe (all three pairs of collectors). We find that our smaller probe $\left(r=0.3 \mathrm{~cm}<\rho_{i}\right)$ has a calibration constant $K=1.9 \pm 0.4$. Our larger probe $\left(r=0.6 \mathrm{~cm}>\rho_{i}\right)$ has a calibration constant $K=2.3 \pm 0.4$.

In figure 3 , we show the average flow in the azimuthal direction as measured by a Mach probe at the location we expect to see the maximum outflow $(r / R \cong 0.8)$. The reconnection outflow in SSX is not purely radial so we expect to see an azimuthal component. Note that the reconnection site isn't coincident with the probe location on every shot making it necessary to average multiple shots together. Flow studies of single spheromaks show that the initial axial flow (up to $100 \mathrm{~km} / \mathrm{s}$ ) returns along the outer wall forming a large scale poloidal vortex. Single spheromaks have very little azimuthal flow or rotation [5].

\section{RECONNECTION HEATING}

Since spheromak merging generates a large $\nabla \times \mathbf{B}$, we expect strong current sheets and direct Ohmic electron heating within the reconnection volume [6]. In addition, the ion flows discussed above would be expected to viscously damp generating a population of heated ions.

\section{A. Electrons}

Model spectra for SSX plasmas have been calculated using an atomic physics code called PrismSpect [12]. Elemental composition has been estimated from vacuum ultraviolet spectroscopy. We observe emission mostly from carbon, with trace emission from oxygen and nitrogen. Soft x-ray (SXR) emission from SSX is measured with a four-channel array of matched PIN photodiodes with thin $(100 \mathrm{~nm})$ metal filters $(\mathrm{Al}, \mathrm{Sn}, \mathrm{Ti}, \mathrm{Zr})$ in front of each. Different filters have different passbands in the range $20-200 \mathrm{eV}$. Using model spectra, we have been able to interpret the SXR flux data to give a dynamical measurement of the electron temperature.

In figure 4, we show the electron temperature calculated from fits of SXR data to model spectra. Note that since the heated electrons are so mobile $\left(v_{e} \cong 2.5 \mathrm{~m} / \mu \mathrm{s}\right)$ they rapidly leave the reconnection volume on newly reconnected field lines and disperse their heat throughout the entire SSX volume. Because of this, the signature of intense electron heating is dissipated in less than $1 \mu \mathrm{s}$. We have noticed that our highest energy x-ray bins have more signal than can be explained with a single temperature electron distribution function. In the future, we plan on modeling spectra using a second, higher temperature electron population or a harder electron tail. Baseline electron temperatures of $T_{e} \cong 20 \mathrm{eV}$ have been independently confirmed by analyzing $C_{I I I} / C_{I V}$ line ratios $(97.7 \mathrm{~nm} / 155 \mathrm{~nm})$ with PrismSpect.

\section{B. Ions}

Ion temperature in SSX merging spheromaks has been measured with the ion Doppler spectrometer at the midplane [9]. Lineshapes from $C_{I I I}$ emission were measured during merging. Ion temperature extracted from the second moment of the lineshape data show heating up to $T_{i} \cong 100 \mathrm{eV}$. Figure 5 shows the ion temperature as a function of time measured at the midplane of SSX. Note that the time of intense ion heating $(30-40 \mu s)$ is the same as the burst of Alfvénic outflow and electron heating noted above. This experiment was performed immediately after a session of He glow discharge conditioning of SSX. We find that our highest $T_{i}$ (and lowest densities) can be attained after the walls have been conditioned to remove adsorbed neutral gas. 


\section{SUMMARY}

Ion and electron dynamics have been measured during reconnection merging events at SSX. Flow integrated along a chord is measured with ion Doppler spectroscopy while local spheromak flow is studied with Mach probes. Electron heating is confirmed via SXR analysis and ion heating is measured with IDS. We observe all these signa- tures (plasma flow, ion and electron heating) coincident with a $3 \mathrm{D}$ magnetic reconnection event at $30-40 \mu s$. Future plans include further studies of energetic electrons using SXR and a new, compact trapezoidal flux conserver for oblate FRC studies.

The authors gratefully acknowledge the technical assistance of S. Palmer and J. Haldeman at Swarthmore, and J. MacFarlane at Prism Computational Sciences.
[1] M. R. Brown, Physics of Plasmas 6, 1717 (1999)

[2] P. M. Bellan, "Spheromaks", Imperial College Press, (2000)

[3] C. G. R. Geddes, T. W. Kornack, and M. R. Brown, Physics of Plasmas 5, 1027 (1998)

[4] C. D. Cothran, A. Falk, A. Fefferman, M. Landreman, M. R. Brown, and M. J. Schaffer, Physics of Plasmas 10, 1748 (2003)

[5] M. R. Brown, et al, Physics of Plasmas 13, 102503 (2006)

[6] M. R. Brown, C. D. Cothran, and J. Fung, Physics of Plasmas 13, 056503 (2006)

[7] M. Landreman, C. D. Cothran, M. R. Brown, M. Kostora, and J. T. Slough, Rev. Sci. Instrum. 74, 2361 (2003)

[8] M. R. Brown, C. D. Cothran, M. Landreman, D. Schlossberg, and W. H. Matthaeus, Astrophysical Journal Lett. $\mathbf{5 7 7}$, L63 (2002)

[9] C. D. Cothran, J. Fung, M. R. Brown, and M. J. Schaffer, Rev. Sci. Instrum. 77, 063504 (2006)

[10] C. D. Cothran, J. Fung, M. R. Brown, and M. J. Schaffer, "Simultaneous Bi-directional Plasma Jets from a Laboratory Magnetic Reconnection Volume", Phys. Rev. Lett. submitted (2007)

[11] I. H. Hutchinson, Physics of Plasmas 9, 1832 (2002)

[12] J. J. MacFarlane, et al, "Inertial Fusion Sciences and Applications", American Nuclear Society, p. 457 (2003)

FIG. 1: Swarthmore Spheromak Experiment. Lines of sight for IDS and probe locations are depicted.

FIG. 2: Outflow jets measured with ion Doppler spectroscopy. Jets persist for several $\mu s$ at $\pm V_{A}$.

FIG. 3: Local Mach probe measurement of outflow. Outflow speed, location, timing $(30-40 \mu s)$ are consistent with IDS data of figure 2 . 
FIG. 4: Electron heating measured with soft x-ray array.

Heating occurs during reconnection activity $30-40 \mu \mathrm{s}$.

FIG. 5: Ion heating measured with IDS. Heating occurs during reconnection activity $30-40 \mu s$. 


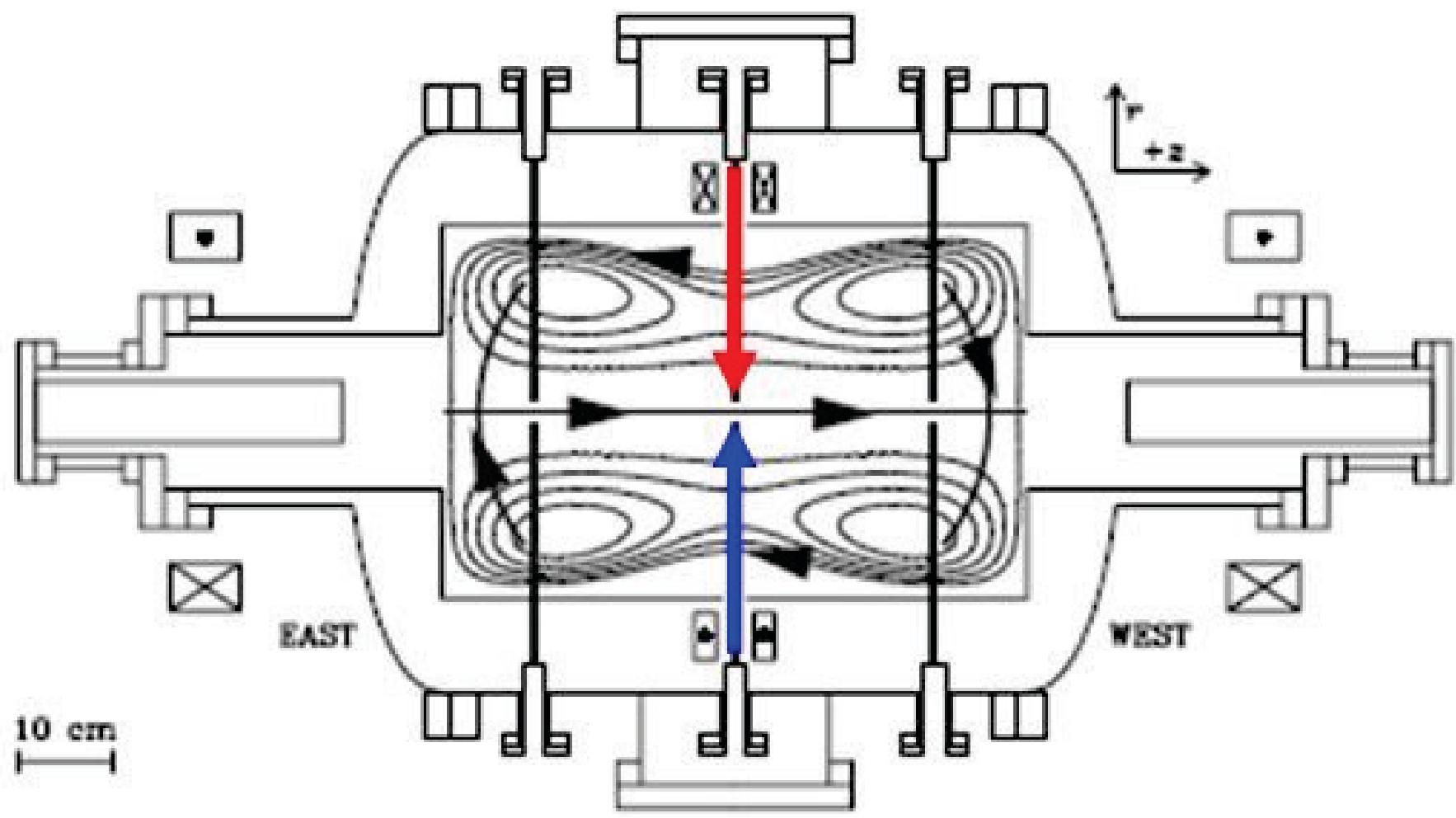




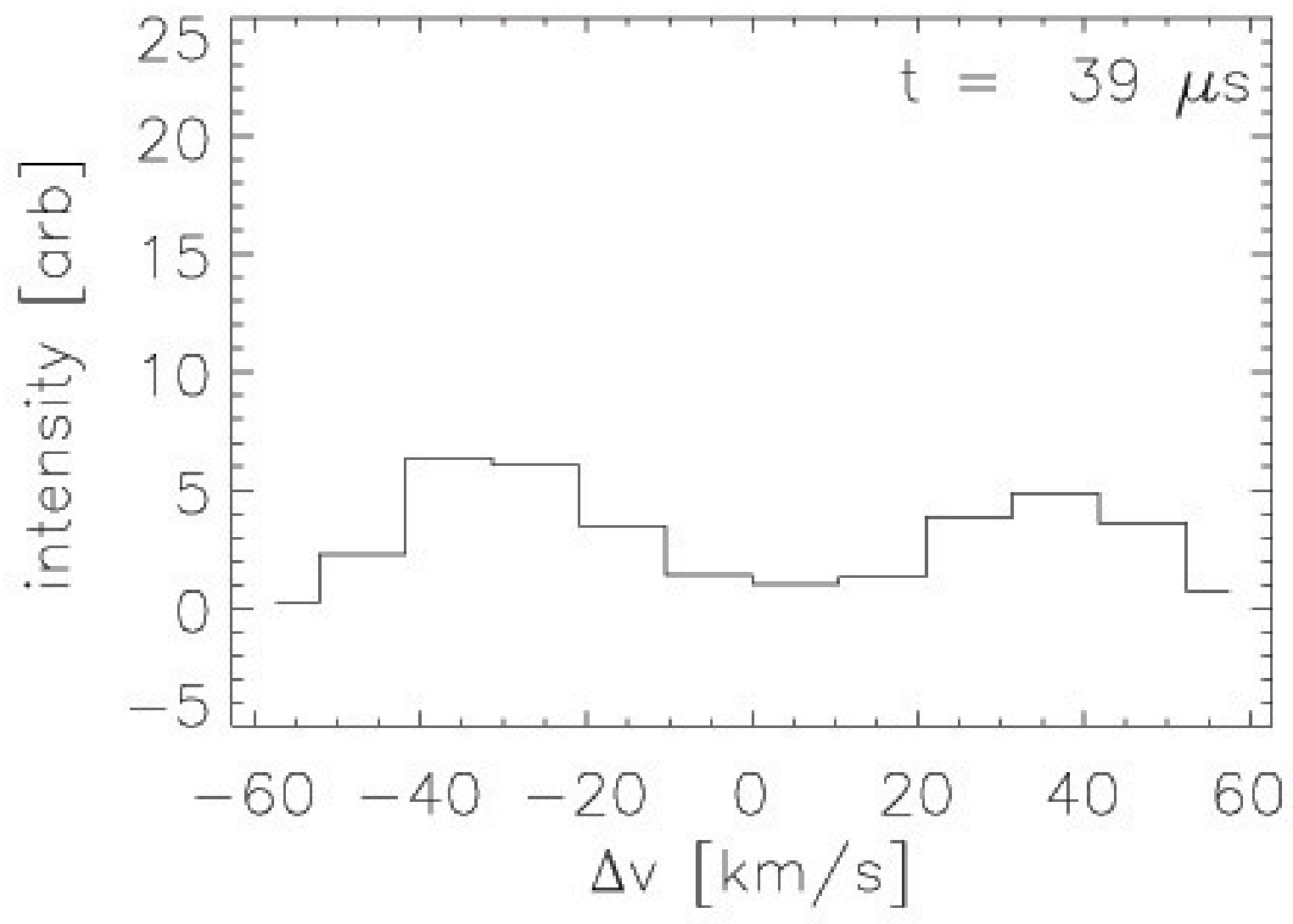


Azimuthal Flows During Counter-Helicity Merging

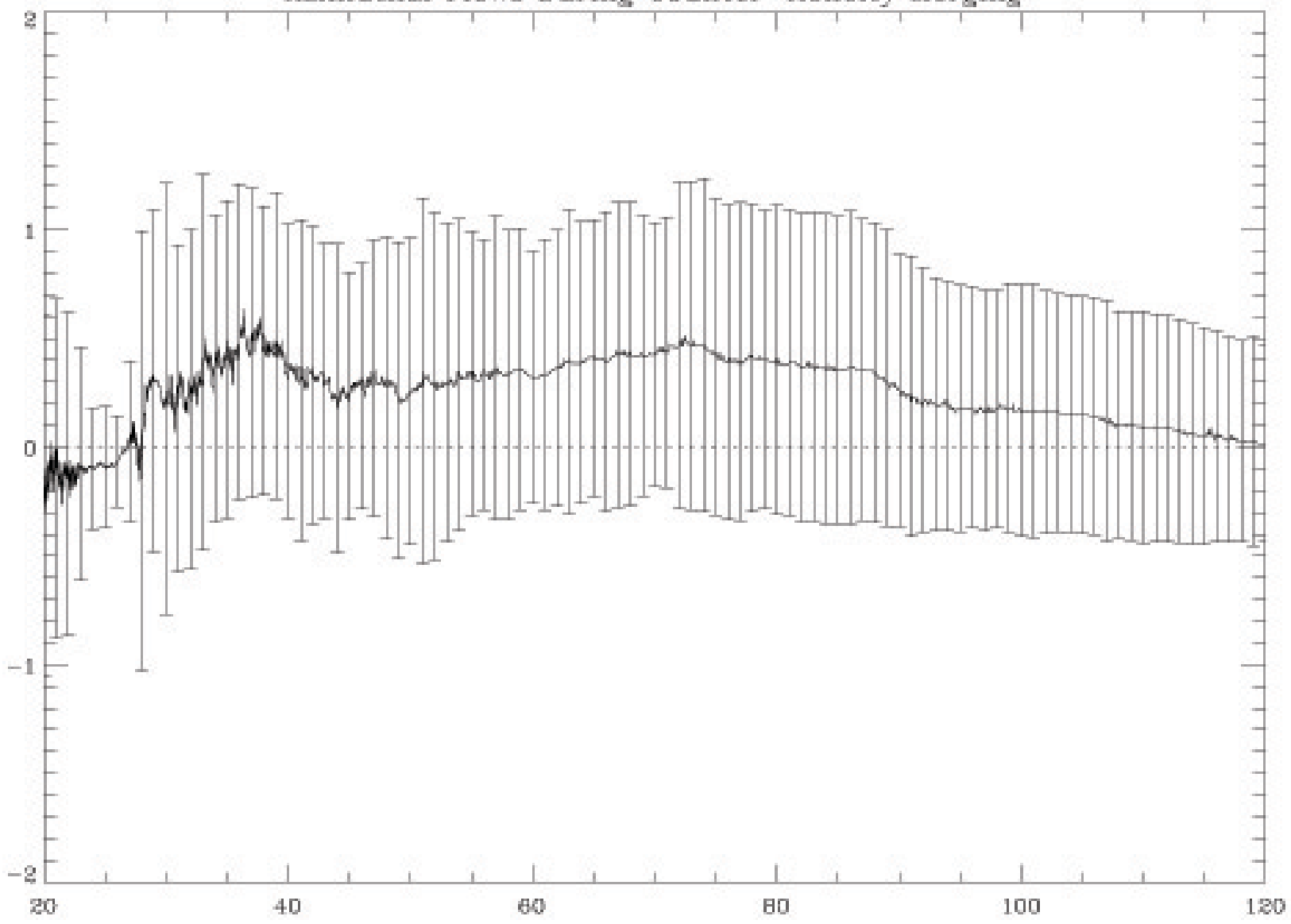




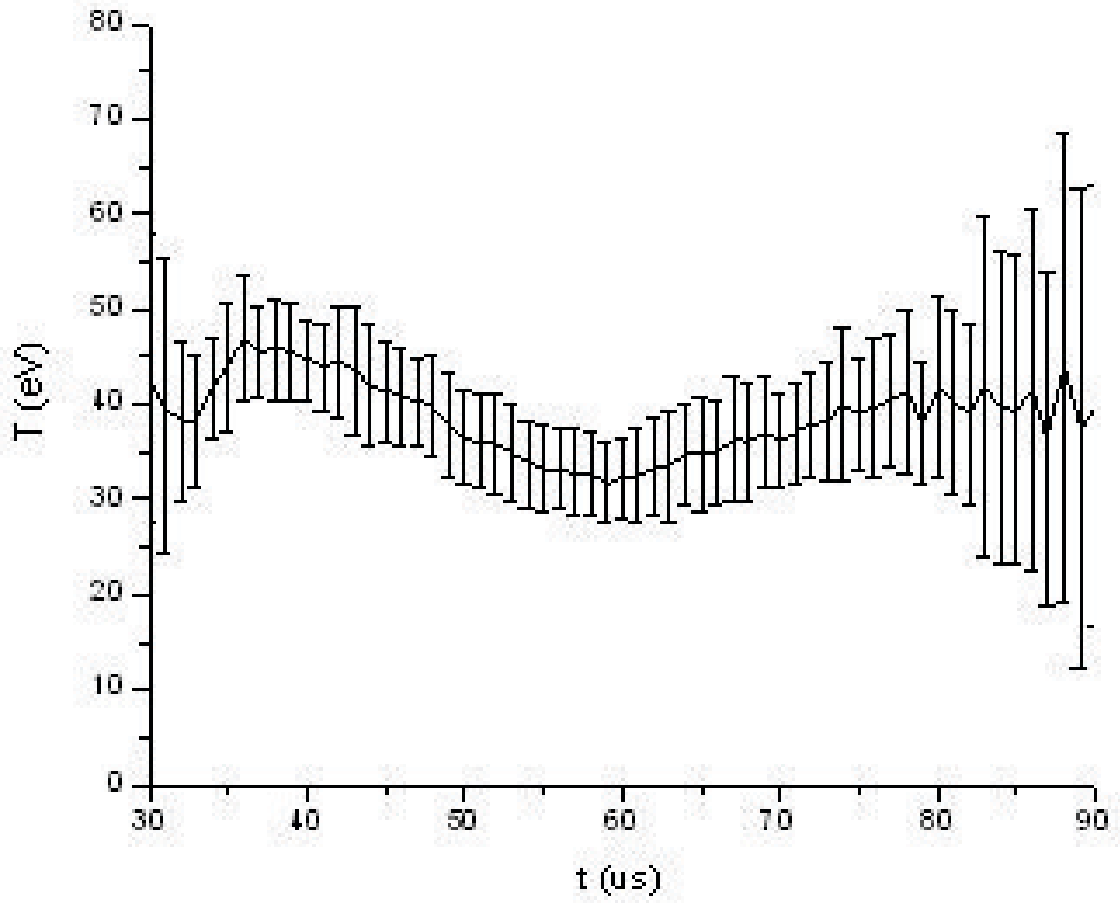




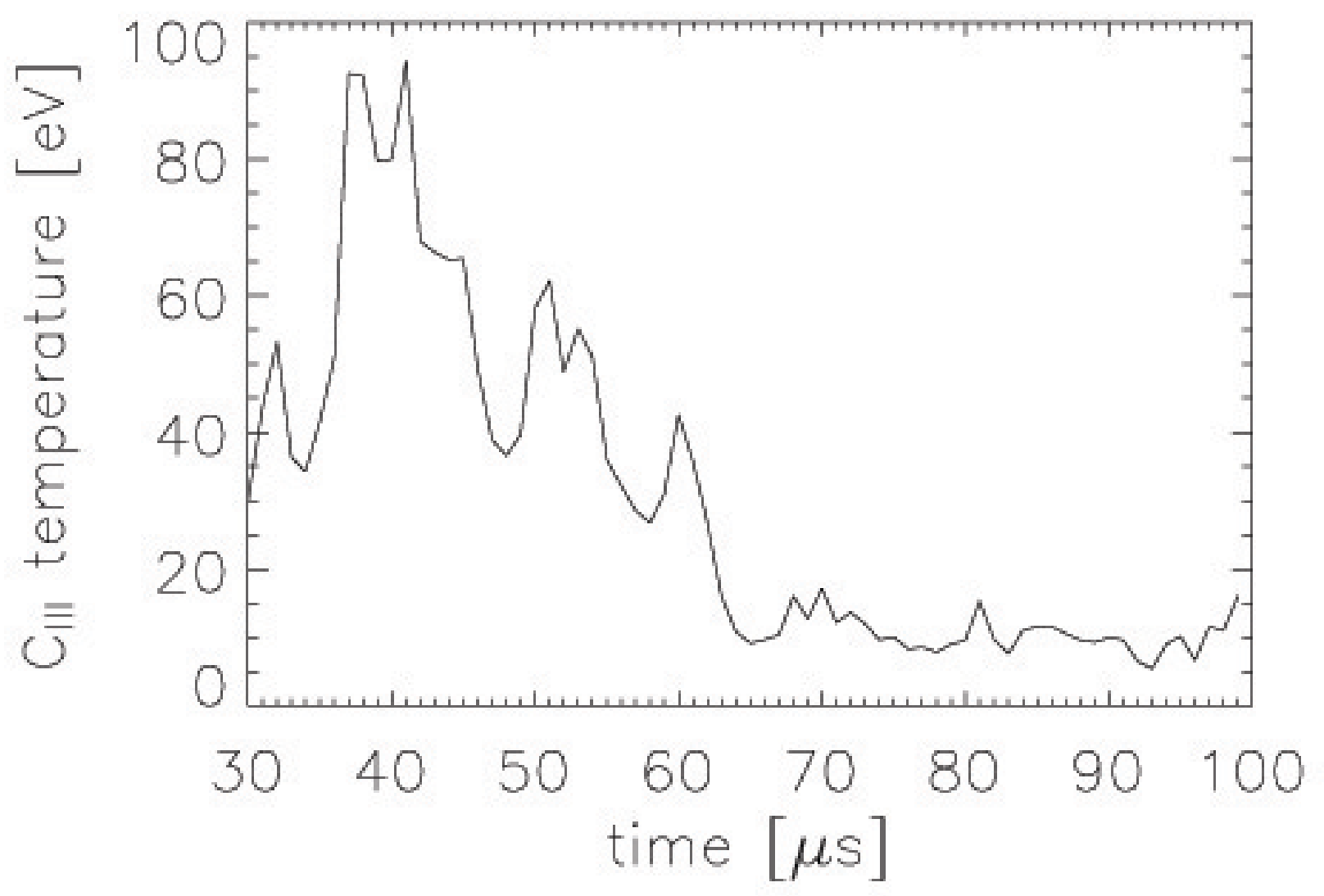

